\title{
Effect of Viscosity on Pumping-Up of Newtonian Fluid Driven by a Rotating Cone*
}

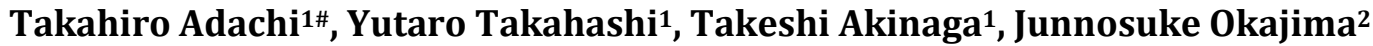 \\ ${ }^{1}$ Department of Systems Design Engineering, Akita University, Akita, Japan \\ ${ }^{2}$ Institute of Fluid Science, Tohoku University, Sendai, Japan \\ Email: "adachi@gipc.akita-u.ac.jp
}

How to cite this paper: Adachi, T., Takahashi, Y., Akinaga, T. and Okajima, J. (2018) Effect of Viscosity on Pumping-Up of Newtonian Fluid Driven by a Rotating Cone. Journal of Flow Control, Measurement \& Visualization, 6, 57-65.

https://doi.org/10.4236/jfcmv.2018.62006

Received: December 4, 2017

Accepted: February 19, 2018

Published: April 12, 2018

Copyright (c) 2018 by authors and Scientific Research Publishing Inc. This work is licensed under the Creative Commons Attribution International License (CC BY 4.0).

http://creativecommons.org/licenses/by/4.0/

\begin{abstract}
Effect of viscosity on flow patterns of pumping-up of liquid generated by a cone rotating at the liquid surface has been experimentally studied with various concentrations of glycerol aqueous solution. We have previously found that the higher viscous non-Newtonian fluid was lifted-up along the conical surface with a radial filament-wise pattern, which is quite different from the monotonic thin film-wise pattern observed for the lower viscous fluid such as water. In order to elucidate the pumping-up mechanism, a transition diagram indicating the critical rotation rate is obtained as a function of viscosity of Newtonian fluid in this study, varying from the lower value of water $(\mu=$ $0.890 \mathrm{mPa} \cdot \mathrm{s})$ to the higher one of glycerin $(\mu=910 \mathrm{mPa} \cdot \mathrm{s})$. It is found that there are three categories depending on the viscosity classified as 1) film-wise pumping-up region for the viscosity $\mu \leq 134 \mathrm{mPa} \cdot \mathrm{s}, 2$ ) filament-wise pumping-up one for the viscosity $\mu \geq 520 \mathrm{mPa} \cdot \mathrm{s}$, and 3) no pumping-up phenomenon occurs for $134<\mu<520 \mathrm{mPa} \cdot \mathrm{s}$.
\end{abstract}

\section{Keywords}

Newtonian Fluid, High Viscosity, Rotating Cone, Pumping-Up, Atomization

\section{Introduction}

In recent atomization devices, new innovations such as energy saving, simplification of maintenance and compactness are strongly required. A centrifugal atomizer that uses the centrifugal force has a potential to achieve the innovations because it is quite compact and simple compared with an existing nozzle based atomizer that uses fans, pumps, etc. as well as compressors for operating it. Only a simple rotating device is necessary for the centrifugal atomizer, where a film

${ }^{*}$ A part of this paper was presented at 14 th International Conference Fluid Dynamics (ICFD14) in Sendai, 1-3 Nov. 2017. 
flow is released from the rotating device and is broken due to instability making liquid droplets.

As a mechanism of the centrifugal atomizer, it is comprehensible and well known that the liquid film flow rises along the inner surface of a rotating hollow cone due to the centrifugal force. Bruin [1] and Makarytchev et al. [2] have studied the phenomena of rising film flow along the inner surface of the rotating hollow cone. They analytically obtained the velocity distribution and film thickness in the liquid film over the rotating conical inner surface. On the other hand, Adachi et al. [3] [4] have found a new atomization technique based on a new mechanism to generate the liquid film flow, which is composed of rotating cone and disk. In the mechanism, liquid is pumped-up along the conical outer surface, where the cone is immersed and rotated in the liquid surface by turning the top upside down. The film flow is generated along the outer surface of the rotating cone and is broken if it goes up enough along the cone surface because it cannot keep the film-wise condition. Eventually, water droplets are generated and atomized into the circumference. This is a quite simple way to make a mist flow composed of homogeneous water droplets. Recently, Adachi et al. [5] have analyzed the film flow thickness along the outer surface of rotating cones by using the boundary layer theory and Rayleigh's criterion.

However, Kuriyagawa and Adachi [6] have shown that the higher viscous non-Newtonian fluid, poly vinyl alcohol (PVA) solution, is pumped-up through the conical outer surface like filament-wise not film-wise. The PVA solution is categorized as a plastic fluid in non-Newtonian Fluid. For such polymer fluid, it is reported that curious phenomena such as Weisenberg effect occur due to the orientation of the non-Newtonian Fluid. Kuriyagawa and Adachi also concluded that the reason of the filamentation of the pimping-up was due to the orientation of the non-Newtonian fluid without detailed explanations.

In this paper, we use a glycerol aqueous solution of Newtonian fluid as higher viscous working fluid because it is easier to investigate the pumping-up phenomena using Newtonian fluid than using non-Newtonian fluid such as PVA. It is expected that the film-wise pumping-up phenomenon is observed for low concentration of glycerol solution with lower viscosity like water, while the filament-wise pumping-up observed for high concentration with higher viscosity of glycerin. Rotation rates for the onset of each pumping-up phenomenon are obtained for varying the concentration of the glycerol aqueous solution from water to glycerin, leading to clarify the transition of flow patterns from film-wise pumping-up to filament-wise one.

\section{Experimental Apparatus}

Figure 1 shows a schematic diagram of the experimental apparatus which generates the pumping-up by a rotating cone. The apparatus is composed of a cone with an apex angle $\theta=60^{\circ}$ (half angle $30^{\circ}$ ) and a bottom diameter of $60 \mathrm{~mm}$, a water tank having the diameter $L=100 \mathrm{~mm}$ and the height $h_{0}=100 \mathrm{~mm}$, a motor, a motor driver, a counter board, a heater, a temperature control device 


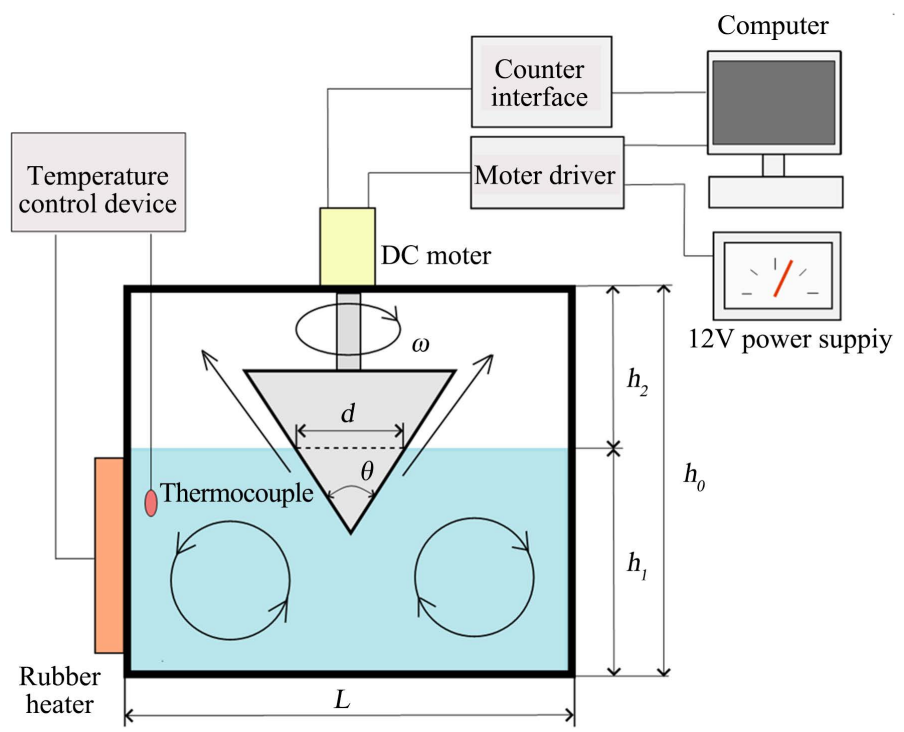

Figure 1. Experimental apparatus.

and a thermocouple as shown in Figure 1. An immersed diameter and a depth in the working fluid are fixed as $d=20 \mathrm{~mm}$ and $h_{1}=55 \mathrm{~mm}$, respectively. The cone made of an Acrylonitrile Butadiene Styrene copolymer is bespoken by a turning process, and no special treatment is given to the surface. The cone is rotated with a rotation rate which is set to a designated value in increments of 100 rpm in a range of 0 to $3500 \mathrm{rpm}$ with the Proportional Integral (PI) control. The temperature of the working fluid is maintained in a range of $25.0^{\circ} \mathrm{C} \pm 0.3^{\circ} \mathrm{C}$ by Proportional Integral Differential (PID) controller.

Water and glycerol aqueous solution are used as working fluids in the present study. The water viscosity is assumed to be $\mu=0.890 \mathrm{mPa} \cdot \mathrm{s}$, whereas the viscosity of glycerol aqueous solution adjusted by the concentration of glycerin is measured by a viscosity meter (Toki industry: TVC-7), where we use the glycerin of $99 \%$ purity and $\mu=910 \mathrm{mPa} \cdot \mathrm{s}$. In this study, in order to clarify the effects of the viscosity on the pumping-up phenomenon, the critical rotation rate when pumping-up occurs is determined as a function of viscosity.

\section{Results}

If a cone rotates on the liquid surface for the larger rotation rate than the critical value $\omega_{\mathcal{c}}$ liquid is lifted up along the cone surface, and we call the phenomenon "pumping-up" by rotating cones. The critical rotation rate when the pumping-up occurs has been measured for glycerol aqueous solutions in a wide range of viscosity. It is confirmed that the critical rotation rate is increased as the viscosity of the fluids is increased from one that is for water as predicted. However, in the higher viscous regime, we newly capture a distinct pumping-up with a very different pattern from one observed for fluids with lower viscosity. Here we shall name the patterns: film-wise and filament-wise pumping-ups for lower and higher viscous fluids, respectively, based on the structure formed on the surface of the cone during pumping-up seen in Figure 2 and Figure 3. 


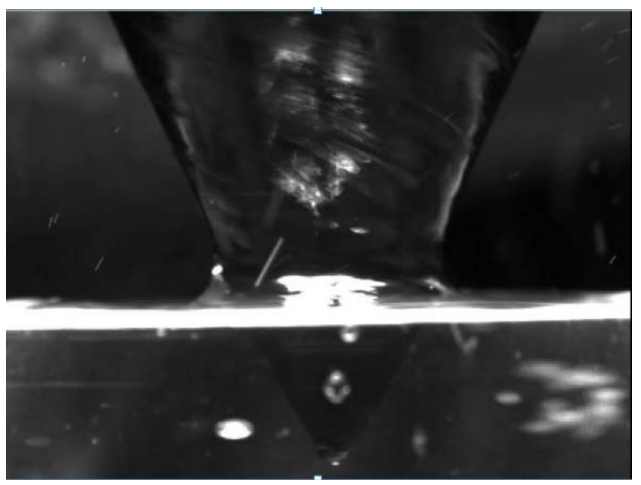

Figure 2. Film-wise pumping-up.

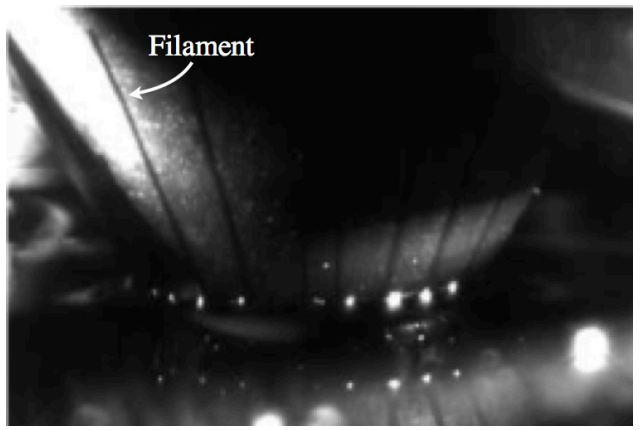

Figure 3. Filament-wise pumping-up.

\subsection{Film-Wise Pumping-Up for Lower Viscous Fluids}

The critical rotation rate $\omega_{c}$ for the film-wise pumping-up is measured as a function of the viscosity $\mu$ of the fluid and is plotted in Figure 4 with circles in red. The pumping-up with film pattern is observed for lower viscosity $\mu \leq \mu_{1}(=134$ $\mathrm{mPa} \cdot \mathrm{s})$. The critical rotation rate $\omega_{c}$ tends to increase as the viscosity is increased from the value for water. For example, $\omega_{c}$ for $\mu=118 \mathrm{mPa} \cdot \mathrm{s}$ is $2700 \mathrm{rpm}$, where for water $(\mu=0.890 \mathrm{mPa} \cdot \mathrm{s}) \omega_{c}$ is $1200 \mathrm{rpm}$. It should be noted, however, that $\omega_{c}$ has a local minimum around $\mu=20 \mathrm{mPa} \cdot \mathrm{s}$. It is not clear the reason why $\omega_{c}$ has the local minimum, but it might be related to the surface tension. Namely, the surface tension is smaller for glycerol aqueous solution than for water. If the surface tension is small, the pumping-up is easily occurred because the surface tension effect works as resistance for the pumping-up. Therefore, if the surface tension is smaller by changing the working fluid from water to glycerol aqueous solution, the critical rotation rate $\omega_{c}$ shows the smaller value. On the other hand, the percentage of the glycerol aqueous solution is increased, the viscosity is increased, which means that the resistance is increased leading to the larger critical rotation rate. The neutral curve becomes steeper as $\mu$ is increased. Note that this phenomenon could not be observed for higher viscosity $\mu>\mu_{1}$ at all, because the upper limitation of the rotation rate in this experimental system is $3500 \mathrm{rpm}$. During the film-wise pumping-up, liquid is covering the entire surface of the cone, and the film-wise structure is almost axisymmetric, i.e., monotonic in the azimuthal direction, as shown in Figure 2. 


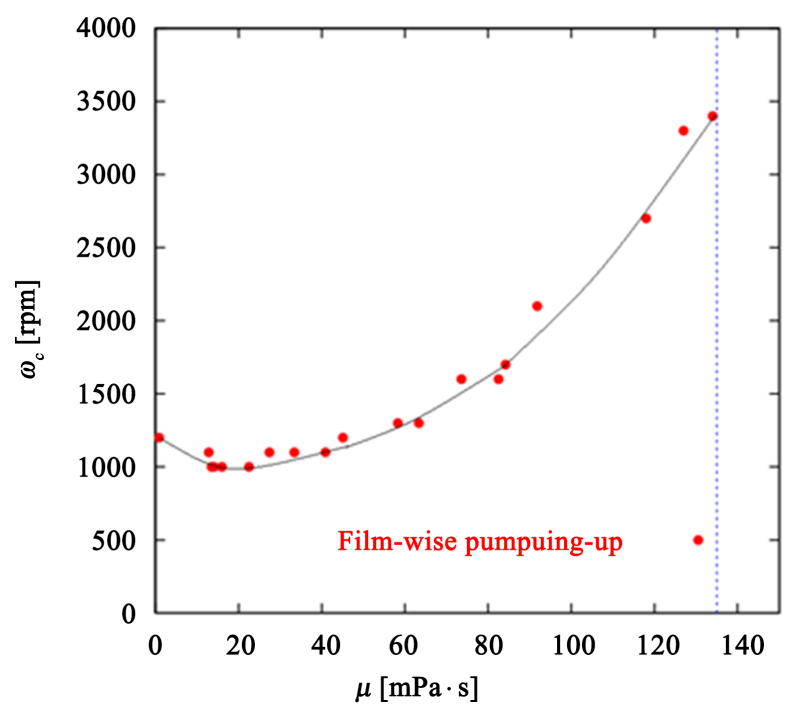

Figure 4. Critical rotation rate, $\omega_{c}$ versus viscosity, $\mu$, for lower viscous fluids.

\subsection{Filament-Wise Pumping-Up for Higher Viscous Fluids}

An apparently different phenomenon compared with the film-wise pumping-up, which is mentioned in the previous subsection 3.1, is observed when we have selected viscous fluids for $\mu \geq \mu_{2}$ (=520 mPa.s). In this range of higher viscosity, the fluid spreads through the radiate filaments formed on the surface of the rotating cone, corresponding to the lines in black shown in Figure 3, and is released to the circumference. By using glycerol aqueous solution, the filament-wise pumping-up is observed for the Newtonian fluids for the first time, and is quantified, while the filament-wise pumping-up has been already observed, qualitatively, in an experimental study [6] with PVA solution which is anon-Newtonian fluid. Blue triangles in Figure 5 indicate the critical rotation rate, $\omega_{\mathcal{c}}$ and the viscosity, $\mu$, when the filament-wise pumping-up occurs. The critical rotation rate decreases with increasing the viscosity, and converges to a constant value (about $2000 \mathrm{rpm}$ ) for viscosity $\mu$ ( $\geq 650 \mathrm{mPa} \cdot \mathrm{s})$.

\section{Discussion}

Film-wise pumping-up phenomenon occurs when glycerol aqueous solution has lower viscosity $\left(\mu \leq \mu_{1}\right)$, and the critical rotation rate is increased as the viscosity is increased. On the other hand, the filament-wise pumping-up phenomenon occurs when the glycerol aqueous solution has higher viscosity $\left(\mu \geq \mu_{2}\right)$, and the critical rotation rate is increased as the viscosity is decreased. For the intermediate viscosity $\left(\mu_{1}<\mu<\mu_{2}\right)$, no pumping-up phenomena has occurred in the present study under the limitation of the maximum rotation rate (3500 rpm) as shown in Figure 6, where the figure is composed of Figure 4 and Figure 5. Further detailed investigation is required for the regime.

In order to comprehend the mechanism of pumping-up phenomena, we herein introduce a small wavy disturbance at the conical surface as depicted in 


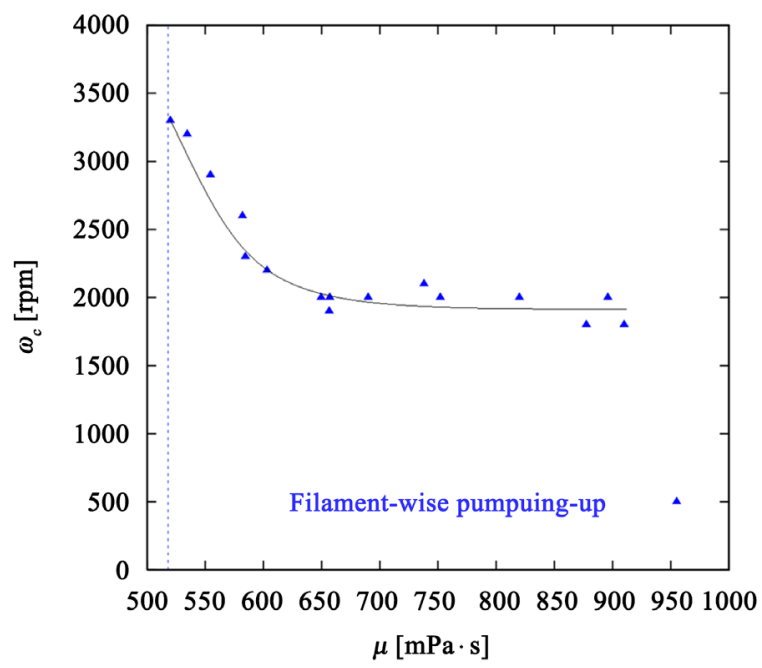

Figure 5. Critical rotation rate, $\omega_{c}$ versus viscosity, $\mu$, for higher viscous fluids.

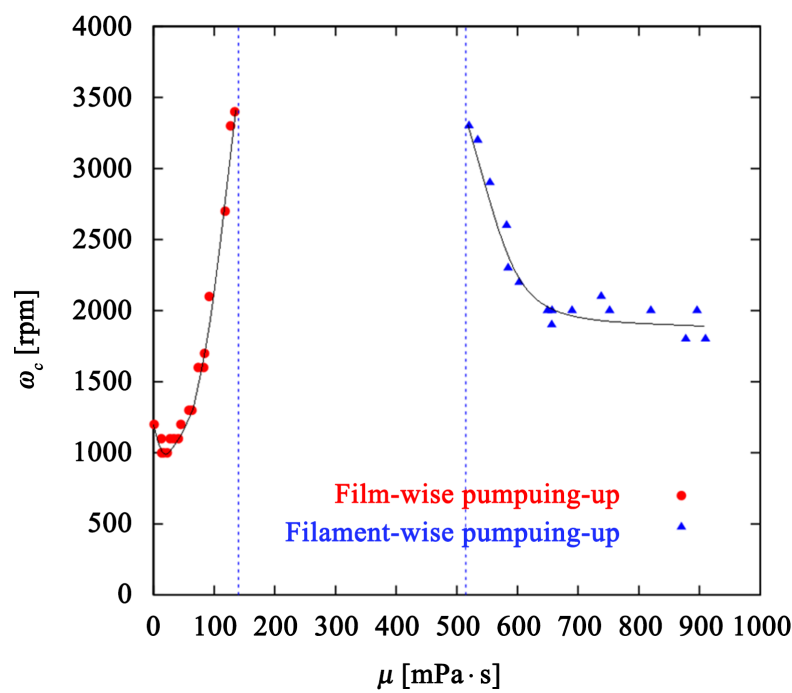

Figure 6. Critical rotation rate, $\omega_{c}$ versus viscosity, $\mu$.

Figure 7(a). The disturbance grows entirely over the conical surface as shown in Figure 7(b), when lower viscous fluid is selected, because of the Rayleigh-Taylor instability due to the centrifugal force field. In other words, the viscous resistance is too small to make the disturbance decay. Therefore, the liquid rises up along the surface in a transient development of the flow field, and the flow becomes a steady state having almost axisymmetric film on the surface. On the other hand, higher viscous fluid $\left(\mu \geq \mu_{2}\right)$ shows other instability: the viscous resistance suppresses the growth of the disturbance, so the growth of the disturbance can be localized at the peaks, i.e., larger radial points, say $r\left(>r^{\prime}\right.$, see Figure $7(c)$ ), on the surface by the larger centrifugal force. As the result of the local growth of the disturbance, filament-wise structures as sketched in Figure 7(d) are selected rather than film-wise structure on the surface in the pumping-up behavior. 


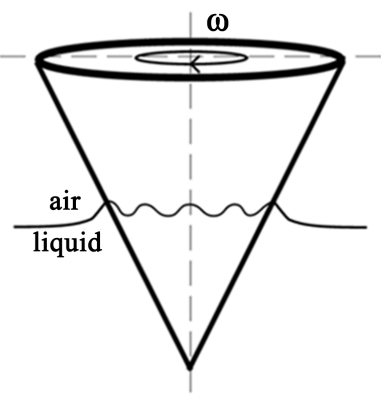

(a)

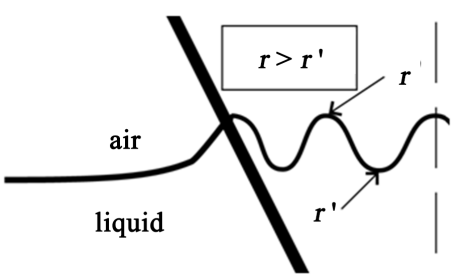

(c)

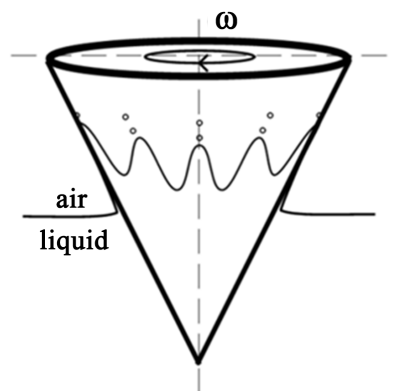

(b)

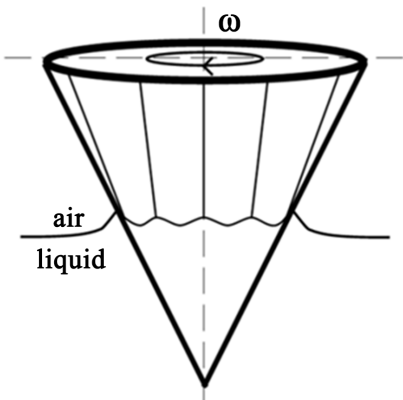

(d)

Figure 7. The occurrence of disturbance due to Rayleigh-Taylor instability. (a) A small wavy disturbance at the conical surface; (b) The growth of disturbance entirely over the conical surface; (c) The growth of the disturbance localized at the peaks of larger radial points as $r>r$; (d) A filament-wise structure.

The critical rotation rate is increased as the viscosity is increased when the film-wise pumping-up occurs. For higher viscosity, the disturbance gets decayed faster, so higher rotation rate for pumping-up would be required in order to grow the disturbance. On the other hand, the critical rotation rate converges to a constant value as the viscosity increases where the filament-wise pumping-up occurs. We speculate about the trend converging to a constant value as follows: the viscous resistance is dominated more as the viscosity is increased, and the liquid cannot rise up along the conical surface. However, if there would exist disturbance far away from the conical surface, the centrifugal force to the disturbance becomes larger because the radius is larger there. Once the centrifugal force overcomes the viscous resistance, the disturbance could grow, resulting in the filament-wise pumping-up. The liquid which starts the pumping-up is strongly influenced by the centrifugal force apart from the cone surface, when the viscosity is higher, whose magnitude might be constant independent of the viscosity. Therefore, it seems that the critical rotation rate converges to a constant value.

\section{Conclusions}

In this paper, we have investigated the transition of the film-wise and filament-wise pumping-up phenomena, where the Newtonian fluid has been ex- 
amined as a working fluid which is composed of glycerol aqueous solution. For varying the concentration of the glycerol aqueous solution from water to glycerin, the viscosity can be changed from the lower value of water to the higher one of glycerin. The critical rotation rates for the onset of each pumping-up phenomenon are obtained for varying the concentration of the glycerol aqueous solution.

It has been shown that there are three categories depending on the viscosity classified as 1) film-wise pumping-up region for the viscosity $\mu \leq 134 \mathrm{mPa} \cdot \mathrm{s}, 2$ ) filament-wise pumping-up region for the viscosity $\mu \geq 520 \mathrm{mPa} \cdot \mathrm{s}$, and 3) no pumping-up phenomena occurs for $134<\mu<520 \mathrm{mPa} \cdot \mathrm{s}$. Furthermore, we have tried to explain the pumping-up mechanism using the concept of Rayleigh-Taylor instability. The film-wise pumping-up phenomenon occurs when the balance between the centrifugal force and viscous one is broken. Namely, under a situation that one component along the cone surface of the centrifugal force from the tip to the bottom is dominant, the heavier fluid of glycerol aqueous solution is located under the lighter fluid of air. If the centrifugal force exceeds the viscous force as well as gravity when the rotation rate increases, the heavier fluid starts to go up. The flow patterns of pumping-up are depending on the viscosity. If the viscosity is lower, the viscous force working on the cone surface is smaller. Then, the working fluid starts to go homogeneously and film-wisely in the azimuthal direction. On the other hand, if the viscosity is higher, the viscous force is also stronger, which leads to non-homogeneous starting of pumping-up like filaments. Unfortunately, we have not observed any pumping-up phenomenon for the intermediate viscosity $\left(\mu_{1}<\mu<\mu_{2}\right)$ mainly due to the limitation of rotation rate arisen from our experimental device.

\section{Acknowledgements}

This work was partially supported by 1) a Grant-in-Aid for Scientific Research (C) 23560185 from The Ministry of Education, Culture, Sports, Science and Technology and 2) Collaborative Research Project of the Institute of Fluid Science, Tohoku University, J17L062. In addition, authors would like to express our thankful heart to Mr. J. Kanamori, Akita University student, for his help in experiment.

\section{References}

[1] Bruin, S. (1969) Velocity Distributions in a Liquid Film Flowing over a Rotating Conical Surface. Chemical Engineering Science, 24, 1647-1654. https://doi.org/10.1016/0009-2509(69)87029-6

[2] Makarytchev, S.V., Langrish, T.A.G. and Prince, R.G.H. (1997) On Modeling Fluid over a Rotating Conical Surface. Chemical Engineering Science, 52, 1055-1057. https://doi.org/10.1016/S0009-2509(96)00473-3

[3] Adachi, T., Sato, N., Kobari, N. and Hori, T. (2010a) Liquid Film Flow Rising along the Outer Surface of the Rotating Cone. The Japan Society of Mechanical Engineering Series B, 76, 161-163. (In Japanese) https://doi.org/10.1299/kikaib.76.761_161 
[4] Adachi, T., Sato, N., Kobari, N. and Hori, T. (2010b) Liquid Film Flow Rising along the Outer Surface of the Rotating Cone. Heat Transfer-Asian Research, 39, 492-496.

[5] Adachi, T., Takahashi, Y. and Okajima, J. (2018) Film Flow Thickness along the Outer Surface of Rotating Cones. European Journal of Mechanics B/Fluids, 68, 39-44. https://doi.org/10.1016/j.euromechflu.2017.11.003

[6] Kuriyagawa, Y. and Adachi, T. (2015) Atomization and Filamentation of High-Viscosity Liquid with a Rotating Cone. Proceedings of 13 th International Conference on Liquid Atomization, Tainan, August 2015.

\section{Nomenclature}

d: immersed diameter

$(\mathrm{mm})$

$h_{0}$ : tank height

$(\mathrm{mm})$

$h_{1}$ : depth of the working fluid

(mm)

$L$ : tank diameter

(mm)

$r$ immersed radius

(mm)

$r$ : larger radial points

(mm)

$\theta$. apex angle of cone

$\left.{ }^{\circ}\right)$

$\mu$ : viscosity

(mPa.s)

$\mu_{1}$ : maximum viscosity of film-wise pumping-up occurrence

$(\mathrm{mPa} \cdot \mathrm{s})$

$\mu_{2}:$ minimum viscosity of filament-wise pumping-up occurrence

$(\mathrm{mPa} \cdot \mathrm{s})$

$\omega_{C}:$ critical rotation rate when pumping-up occurs

(rpm) 